\title{
Risk of Late Mortality and Second Malignant Neoplasms among 5-Year Survivors of Young Adult Cancer: A Report of the Childhood, Adolescent, and Young Adult Cancer Survivors Research Program
}

\author{
Yang Zhang, ${ }^{1,2}$ Karen Goddard, ${ }^{3}$ John J. Spinelli, ${ }^{1,2}$ \\ Carolyn Gotay, ${ }^{1,2}$ and Mary L. McBride ${ }^{1}$ \\ ${ }^{1}$ Cancer Control Research Program, British Columbia Cancer Research Centre, British Columbia Cancer Agency, Vancouver, \\ BC, Canada V5Z 1 L3 \\ ${ }^{2}$ School of Population and Public Health, Faculty of Medicine, University of British Columbia, Vancouver, BC, Canada V6T $1 Z 3$ \\ ${ }^{3}$ Division of Radiation Oncology, BC Cancer Agency, Vancouver, BC, Canada V5Z 4E6
}

Correspondence should be addressed to Mary L. McBride, mmcbride@bccrc.ca

Received 28 April 2012; Revised 23 July 2012; Accepted 8 August 2012

Academic Editor: Paul Nathan

Copyright ( 2012 Yang Zhang et al. This is an open access article distributed under the Creative Commons Attribution License, which permits unrestricted use, distribution, and reproduction in any medium, provided the original work is properly cited.

\begin{abstract}
We conducted a population-based retrospective study to assess the long-term risks of overall and cause-specific mortality and second malignant neoplasm (SMN) among survivors of young adult cancer compared to the risk in British Columbia (BC) population and to evaluate the effects of demographic and clinical factors on risk. 12485 -year survivors of young adult cancer diagnosed 1970-1995 between 20 and 24 years of age were identified from the BC Cancer Registry and followed to the end of 2007. Standardized mortality ratios (SMRs) and standardized incidence ratios (SIRs) were calculated. The Cox proportional hazards model was used to estimate the effects of different demographic and disease-related characteristics on the risk of death and SMN. A total of 138 deaths and 62 SMNs were observed during follow-up. The overall SMR was 5.9 (95\% CI 4.9-6.9) and the absolute excess risk was 5.3 per 1,000 person-years. The overall SIR was 3.0 (95\% CI 2.3-3.8). Treatment with radiation resulted in increased risks of death and SMN. These observed increased risks emphasize the importance of prevention, surveillance, and treatment of late effects in survivors of young adult cancers.
\end{abstract}

\section{Introduction}

Advances in therapy for young adult cancers have led to an increase in the relative 5 -year survival rate from $71 \%$ in 1975 to $87 \%$ in 2007 [1]. This has created a growing population of young adult cancer survivors. In Canada, presently more than 150,000 Canadians are survivors of a young adult cancer diagnosed since 1980 [2]. Long-term survivors of young adult cancer may face serious health risks including an increased risk of a second malignant neoplasm (SMN), excess late mortality, and functional impairment of multiple organ systems $[3,4]$.

Previous studies have shown that survivors of childhood and adolescent cancer have increased mortality risks, compared with the general population [5-10]. Death in these survivors is mainly due to the original cancer, followed by deaths due to second malignant neoplasm (SMN) and noncancer causes $[5,7,8,11,12]$.

Long-term survivors of childhood and adolescent cancer are also at risk for the development of an SMN. This risk is likely to be the consequence of multiple factors, including environment factors and genetic susceptibility related to the original diagnosis, the treatment used, immunosuppression, and hormonal factors [3]. Previous studies have reported that the relative risk of developing SMN varies between 2.4 and 6.1 in this group [13], depending on characteristics of the study population, the types of SMN considered, the diagnosis time period, and the length of follow-up time included in the analyses. However, there is little information on late mortality and SMN risks specifically among young adult cancer survivors. 
The Childhood, Adolescent and Young Adult Cancer Survivors (CAYACS) Research Program utilizes record linkage methodology and data from population-based registries, administrative databases, and medical charts, to examine issues among survivors of cancer diagnosed under age of 25 years in British Columbia (BC), Canada [14]. Since a review of the available literature indicated that there was little published information on long-term mortality and SMN risks for survivors of young adult cancer, we conducted a population-based retrospective study to assess the longterm risks of overall and cause-specific mortality and SMN among survivors of young adult cancer in the CAYACS cohort, as compared to these risks in the British Columbia population. We also evaluated the demographic and clinical factors affecting these risks.

\section{Materials and Methods}

2.1. Study Population. The CAYACS program's design and methodology has been described in detail elsewhere [14]. In brief, the cancer survivor cohort was identified from the BC Cancer Registry (BCCR). The Registry receives notice of all cancer diagnoses occurring in Canada to BC residents, via multiple sources of ascertainment [14]. In this study, all subjects had a first diagnosis of cancer between 20 and 24 years of age included in the Adolescent and Young Adult (AYA) Cancer Classification [15], excluding non-melanoma skin cancer and surviving at least five years from diagnosis. All survivors were residents in BC at time of diagnosis, diagnosed between January 1, 1970 and December 31, 1995, and followed to the end of 2007.

\subsection{Data Collection}

\subsubsection{Outcome Information}

(1) Death Information. Death records are maintained at the Ministry of Health Vital Statistics Agency (VSA) and routinely reported to the BCCR within 18 months of the death year. Information documenting death, including date and original causes of death code using the International Classification of Disease version 9 (ICD-9) [16], was obtained from the BCCR. Survivors without death information were considered alive at the end of the followup period. The sex-, age-, cause-, and year-specific mortality rates for the BC population were obtained from the VSA. Specific causes of death were combined into three major groups: (1) death due to recurrence or progression of the original cancer (ICD 140-239); (2) death due to a second original cancer (ICD 140-239); (3) death due to noncancerrelated cause, including disease-related causes and external causes (ICD 001-139, 240-999).

(2) Second Malignant Neoplasm (SMN). SMNs were ascertained from the BCCR. As defined in this study, SMN was (1) a neoplasm that occurred between 5 years after the original diagnosis and December 31, 2007, (2) a diagnosis included in the International Classification of Disease for Oncology version 3 (ICDO-3) [17] with a behavior code of 3 or higher, indicating a malignant tumor, and (3) in a new location or in the same location with a new histology code. Benign tumors, local recurrence, and metastases from the original cancer were not considered as SMNs. Only the first subsequent neoplasm occurring more than 5 years after the original diagnosis was counted as an SMN in this study; thus survivors who developed a second malignant neoplasm within the first 5-year after the original diagnosis were removed from the analysis $(n=9)$.

2.2.2. Modifying Variables. Demographic characteristics, diagnostic information on the original cancer, and follow-up information were collected from the BCCR. Medical records from tertiary cancer centers in $\mathrm{BC}$ were abstracted to obtain clinical information, including whether or not the survivors received surgery, chemotherapy or radiation therapy (RT) for the original cancer, and the location of the radiation therapy. Approximately $72 \%$ of subjects were referred to a tertiary cancer center [14]. As all RT facilities in the province are located in a tertiary cancer center, the survivors' RT records were considered complete, but information regarding chemotherapy and surgery was missing for those cases not referred to any of these centers, as we did not have access to information for cases treated outside this system.

2.3. Statistical Analysis. Frequency distributions and differences in demographic and clinical characteristics across outcome groups were calculated.

The expected numbers of deaths, standardized mortality ratios (SMRs), and absolute excess risks (AERs) of the young adult survivor cohort were estimated by type of original diagnosis, diagnosis period, length of follow-up, type of treatment, and major cause of death. Person-years at risk were calculated as the time from 5 years after the original cancer diagnosis to death or the end of follow-up period (December 31, 2007). The expected number of deaths was computed by multiplying the sex-, age-, cause-, and yearspecific mortality rate in $\mathrm{BC}$ by the person-years at risk in each category in the study group. The SMR was calculated as the number of observed deaths divided by the expected number. The AER of death was calculated as the observed number of deaths minus the expected number, divided by the person-years at risk, and reported as per 1,000 cancer survivors per year.

Standardized incidence ratios (SIRs) and related AERs were calculated using the same methods. Person-years at risk for SMN were calculated from 5 years post original cancer diagnosis to diagnosis of the first SMN, death, or the end of the follow-up.

The cumulative incidence function for both mortality and SMN was estimated for each sex. The Cox proportional hazards regression model was used to estimate the effects of different survivor- (age and sex), disease- (e.g., type and period of diagnosis of the original cancer, having early relapse), and treatment-related characteristics (chemotherapy, RT, and surgery) on the risks of death and SMN. In addition, the method developed by Fine and Gray was also applied in the analysis of SMN to estimate subdistribution 
hazard ratios (HRs) by considering death as a competing risk [18]. Since these two methods gave very similar results for effects of SMN risk factors, only the results of subdistribution hazard ratios were presented.

For missing chemotherapy and surgery status on cases who were not referred to any of the tertiary cancer center, multiple imputation was utilized under the assumption of missing at random [19]. The imputation model contained information on sex, type of original cancer (seven categories in the mortality analysis, five categories in the SMN analysis), diagnosis period (10-year group), having relapse $<5$ years after diagnosis (yes/no), and having radiation (yes/no), and created 20 datasets without missing chemotherapy or surgery value. The analysis was repeated 20 times and the results were combined and summarized to generate valid statistical inferences. All calculations were performed using the statistical package SAS version 9.2 (SAS Institute Inc, Cary, NC, USA).

2.4. Study Approvals. The study received ethical approval from the University of British Columbia/BC Cancer Agency Clinical Research Ethics Board. Approval for access, use, and linkage of data was obtained from the BC Cancer Registry and the BC Cancer Agency Health Records Departments. To protect confidentiality, all counts less than 5 were masked in tables and text.

\section{Results}

3.1. Descriptive Analysis. In total, 1248 survivors were identified, including $589(47.2 \%)$ males and $659(52.8 \%)$ females. The survivors were followed to the end of 2007, more than 20 years on average after their original cancer diagnosis ( mean $=23.4$ years for the mortality study; mean $=23.1$ years for the SMN study). Among the survivors in the cohort, 27\% ( $n=341)$ were diagnosed with carcinoma, including cancers in the thyroid $(n=117)$ and cervix and uterus $(n=92), 25 \%$ had lymphoma $(n=307)$, and $18 \%$ had melanoma $(n=$ 222) (Table 1). About a third (31.6\%) were diagnosed before 1980. In total, 369 (29.6\%) young adult cancer survivors received RT as a component of their treatment, and 342 (out of 894 with known chemotherapy status, $38.3 \%$ ) survivors received chemotherapy.

3.2. Late Mortality. After 21711 person-years of follow-up, a total of 138 deaths (138/1248 or $11.1 \%$ of the cohort) were observed more than 5 years after diagnosis (Table 2). Of these, 117 deaths $(84.8 \%)$ had cause of death information. There were 16,835 person-years beyond 20 years after the original diagnosis, and 18,689 person-years beyond age 40 years. The mean age at the end of follow-up was 46.0 (SD \pm 7.2 ), and the mean duration of follow-up from diagnosis was 23.4 years $(\mathrm{SD} \pm 7.1)$.

The overall mortality rate for the young adult survivors was almost 6 times higher than the corresponding rate for the BC population (SMR $=5.9 ; 95 \% \mathrm{CI}, 4.9-6.9)$ (Table 2). Survivors of all original diagnoses measured had elevated mortality compared to the $\mathrm{BC}$ population rate. Central

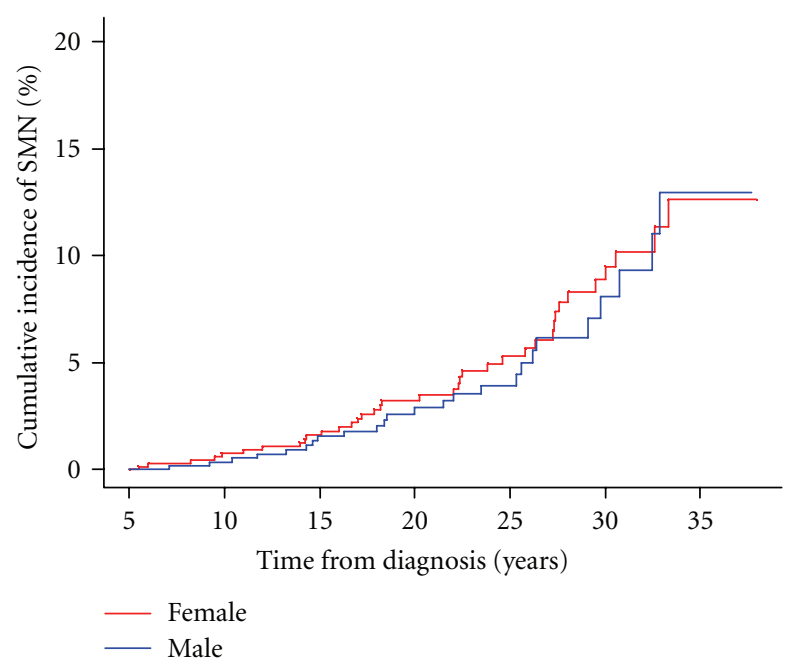

Figure 1: Cumulative incidence of SMN by sex.

nervous system (CNS) tumor survivors had the highest SMR (SMR $=23.6 ; 95 \% \mathrm{CI}, 15.1-35.1)$. The smallest excess risks were seen in subjects originally diagnosed with germ cell tumor or carcinoma and having a longer time of follow-up. Overall, females (SMR $=6.3 ; 95 \%$ CI, 4.8-8.3) had a higher risk of death compared to males (SMR $=5.6$; 95\% CI, 4.56.9).

Among the 117 deceased cases with known cause of death, death due to original cancer was the leading cause, with $62(53.0 \%)$ cases attributed to the recurrence or progression of the original cancer diagnosis, representing a crude rate of 2.86 per 1,000 person-years (not shown in table). An additional 23 (19.7\%) deaths were attributed to a different cancer, and $32(27.4 \%)$ deaths were reported as due to noncancer-related causes, including external causes, such as accident and suicide. Male survivors showed higher risk of death due to SMN and noncancer causes than females. For cumulative mortality, male survivors had higher risk over time than females (Figure 2). Of the 98 deaths followed less than 20 years (87 cases with known causes of death), the leading cause of death was original cancer (56/87 deaths, $64.4 \%$, not shown in table), followed by noncancer death (19/87 deaths, $21.8 \%)$. For survivors who had survived at least 20 years ( 30 cases with known cause of death), deaths due to a cause other than cancer (13 of 30 deaths, $43.3 \%$ ) and SMNs (10 of 30 deaths, $33.3 \%$ ) were the two most common causes of death.

3.3. Second Malignant Neoplasm. Of the 1248 survivors of young adult cancer in the study, 62 developed an SMN during the follow-up period (Table 3 ). The average latency period between cohort entry and diagnosis of the SMN was 19.9 years with a cumulative incidence of $5.0 \%(95 \% \mathrm{CI}$, $3.5 \%-6.6 \%) 20$ years after study entry. Of the 62 cases who had an SMN, 37.1\% $(n=23)$ were deceased by the end of follow-up. The two most common SMNs were breast carcinoma $(n=18)$ among female survivors, which was 
TABLE 1: Characteristics of young adult cancer survivors by sex.

\begin{tabular}{|c|c|c|c|}
\hline Characteristic & Entire cohort $N(\%)$ & Male $N(\%)$ & Female $N(\%)$ \\
\hline Overall & 1248 & $589(47.2)$ & $659(52.8)$ \\
\hline \multicolumn{4}{|l|}{ Type of original cancer } \\
\hline Lymphoma & $307(24.6)$ & $162(27.5)$ & $145(22)$ \\
\hline Central nervous system tumor & $59(4.7)$ & $36(6.1)$ & $23(3.5)$ \\
\hline Soft tissue sarcoma & $66(5.3)$ & $40(6.8)$ & $26(4.0)$ \\
\hline Germ cell tumor & $178(14.3)$ & $161(27.3)$ & $17(2.6)$ \\
\hline Melanoma & $222(17.8)$ & $80(13.6)$ & $142(21.5)$ \\
\hline Carcinoma & $341(27.3)$ & $71(12.1)$ & $270(41)$ \\
\hline Others $^{\mathrm{a}}$ & $75(6.0)$ & $39(6.6)$ & $36(5.5)$ \\
\hline \multicolumn{4}{|l|}{ Birth year } \\
\hline $1945-1954$ & $271(21.7)$ & $115(19.5)$ & $156(23.7)$ \\
\hline $1955-1964$ & $528(42.3)$ & $261(44.3)$ & $267(40.5)$ \\
\hline $1965-1975$ & $449(36)$ & $213(36.2)$ & $236(35.8)$ \\
\hline \multicolumn{4}{|l|}{ Diagnosis period } \\
\hline $1970-1979$ & $394(31.6)$ & $170(28.9)$ & $224(34.0)$ \\
\hline 1980-1989 & $520(41.7)$ & $259(44)$ & $261(39.6)$ \\
\hline 1990-1995 & $334(26.8)$ & $160(27.2)$ & $174(26.4)$ \\
\hline \multicolumn{4}{|l|}{ Attained age (years) } \\
\hline $25-39$ & $366(29.3)$ & $179(30.4)$ & $187(28.4)$ \\
\hline $40-49$ & $522(41.8)$ & $265(45)$ & $257(39.0)$ \\
\hline $50+$ & $360(28.8)$ & $145(24.6)$ & $215(32.6)$ \\
\hline \multicolumn{4}{|l|}{ Follow-up time (years) } \\
\hline $5-19$ years & $521(41.7)$ & $250(42.4)$ & $256(38.8)$ \\
\hline $20+$ years & $727(58.3)$ & $339(57.6)$ & $403(61.2)$ \\
\hline \multicolumn{4}{|l|}{ Relapse $<5$ years after diagnosis } \\
\hline Yes & $82(6.6)$ & $46(7.8)$ & $36(5.5)$ \\
\hline No & $1166(93.4)$ & $543(92.2)$ & $623(94.5)$ \\
\hline \multicolumn{4}{|l|}{ Vital status } \\
\hline Dead & $138(11.1)$ & $84(14.3)$ & $54(8.2)$ \\
\hline Alive & $1110(88.9)$ & $505(85.7)$ & $605(91.8)$ \\
\hline \multicolumn{4}{|l|}{$\mathrm{SMN} \geq 5$ years after diagnosis } \\
\hline Yes & $62(5)$ & $25(4.2)$ & $37(5.6)$ \\
\hline No & $1186(95)$ & $564(95.8)$ & $622(94.4)$ \\
\hline \multicolumn{4}{|l|}{ Having chemotherapy } \\
\hline Yes & $342(27.4)$ & $200(34)$ & $142(21.5)$ \\
\hline No & $552(44.2)$ & $267(45.3)$ & $285(43.2)$ \\
\hline Missing & $354(28.4)$ & $122(20.7)$ & $232(35.2)$ \\
\hline \multicolumn{4}{|l|}{ Having radiation } \\
\hline Yes & $369(29.6)$ & $204(34.6)$ & $165(25.0)$ \\
\hline No & $879(70.4)$ & $385(65.4)$ & $494(75.0)$ \\
\hline \multicolumn{4}{|l|}{ Having surgery } \\
\hline Yes & $628(50.3)$ & $332(56.4)$ & $296(44.9)$ \\
\hline No & $270(21.6)$ & $137(23.3)$ & $133(20.2)$ \\
\hline Missing & $350(28.0)$ & $120(20.4)$ & $230(34.9)$ \\
\hline
\end{tabular}

${ }^{a}$ Other types of original cancers include leukemia, bone tumor, and miscellaneous-specified tumor.

most often after a lymphoma diagnosis $(n=10)$, and cancer of digestive system among males $(n=9)$.

Young adult cancer survivors had a 3 -fold higher rate of SMN (SIR $=3.0,95 \% \mathrm{CI}, 2.3-3.8)$ compared to the cancer incidence rate of the $\mathrm{BC}$ population (Table 3 ). Lymphoma survivors were 7 times more likely to experience an SMN (SIR $=7.0,95 \%$ CI, 4.7-9.9). The SIR decreased with time, from $11.6(95 \% \mathrm{CI}, 7.9-16.6)$ in the period 5-19 years later, to 1.7 (95\% CI, 1.2-2.5) in the period $20+$ years from diagnosis. It increased from 2.5 to 4.0 for survivors diagnosed 


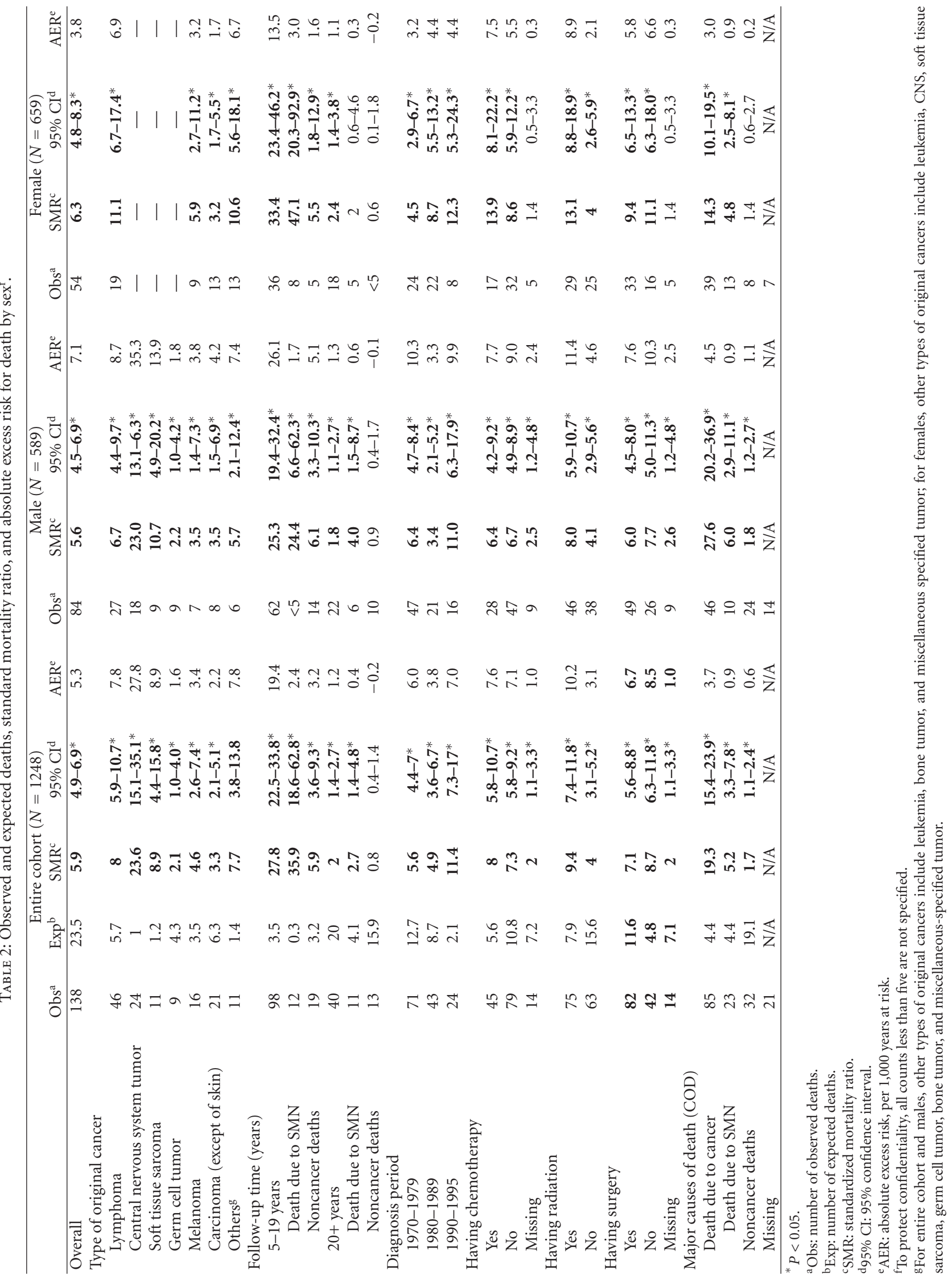




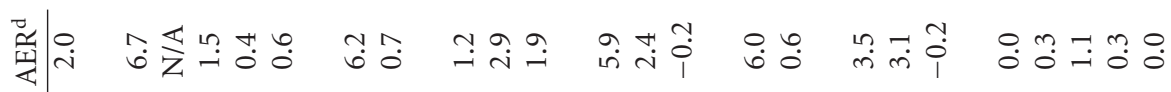

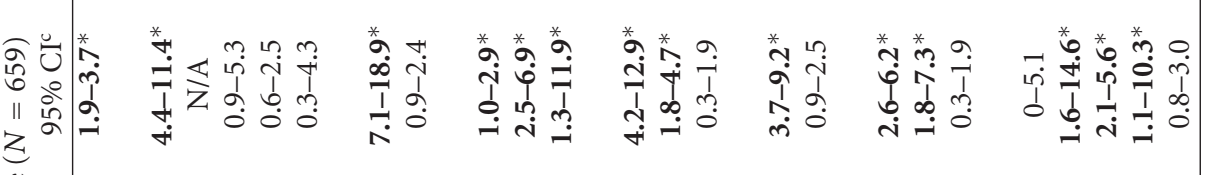

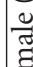

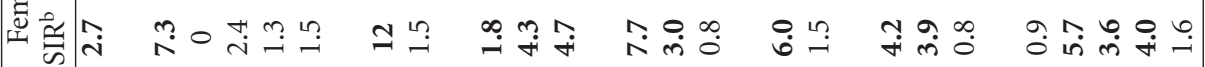

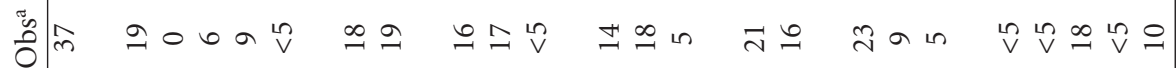

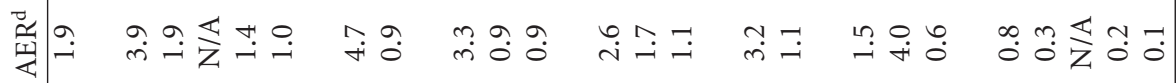

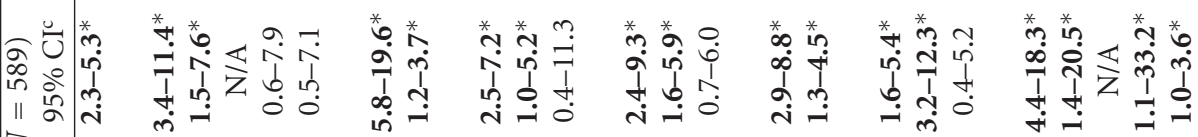

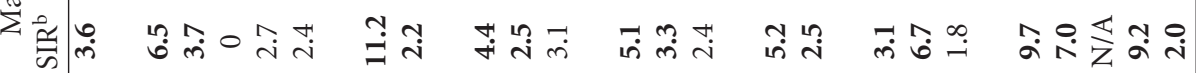

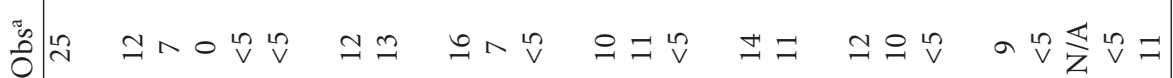

爱

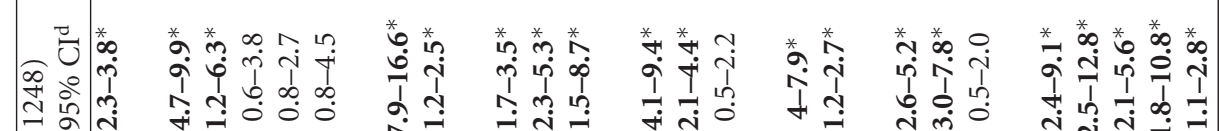

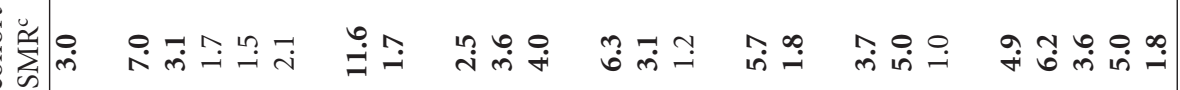
昙

商

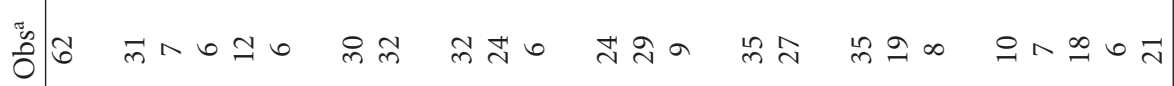

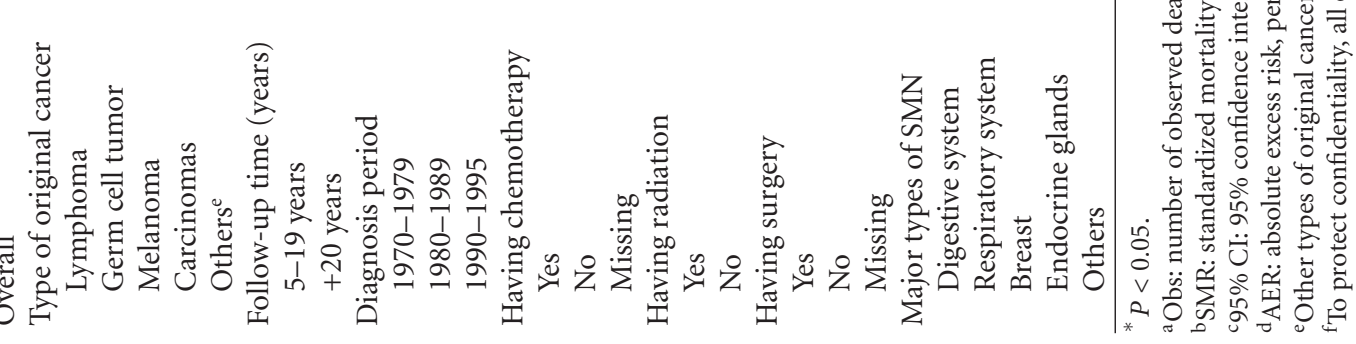




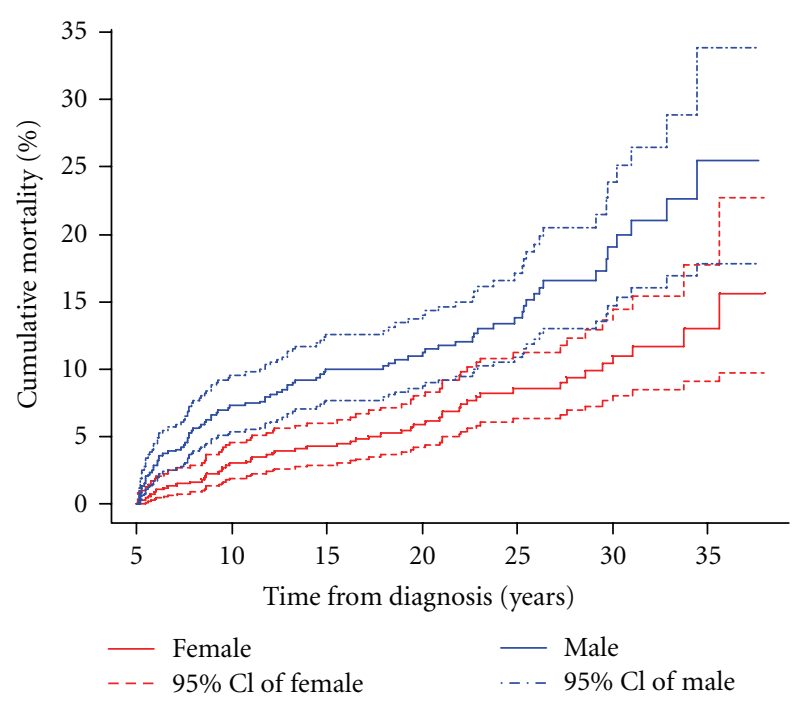

FIgure 2: Cumulative mortality by sex.

in the 1970s compared to those diagnosed in the early 1990s. The cumulative incidence of developing an SMN for both sexes did not show significant difference over time (Figure 1).

RT increased the risk of SMN (SIR, 5.7 versus SIR, 1.8 for patients without radiation exposure). Of the 35 SMNs in survivors who received RT, 19 (54.3\%) developed an SMN within the previous radiation field (as determined by the radiation oncologist), most following RT for Hodgkin lymphoma (16 of 19). Twenty-six of 31 SMN cases with an original diagnosis of Hodgkin lymphoma had received RT. Twenty-seven survivors developed the SMN in the absence of radiation exposure, including 15\% (4 of 27) treated with chemotherapy, $52 \%$ (14 of 27) without chemotherapy, and 9 cases with missing chemotherapy data.

3.4. Factors Affecting Late Mortality and SMN Risk. Table 4 shows the unadjusted and adjusted associations between characteristics of survivors of young adult cancer, and the likelihood of death during the follow-up period. After adjusting for other factors, survivors of CNS had a statistically significant increased risk of dying (Hazard Ratio $\left.[\mathrm{HR}]_{\mathrm{adj}}=3.4,95 \% \mathrm{CI}, 2.1-5.7\right)$ compared to lymphoma survivors, whereas germ cell tumor and carcinoma survivors had decreased risks $\left(\mathrm{HR}_{\mathrm{adj}}=0.4,95 \% \mathrm{CI}, 0.2-0.7 ; \mathrm{HR}_{\mathrm{adj}}=\right.$ $0.5,95 \%$ CI, $0.3-0.8$, resp.). Male survivors were 1.7 times more likely to die than their female counterparts $\left(\mathrm{HR}_{\mathrm{adj}}=\right.$ 1.7, 95\% CI, 1.2-2.4). Five-year survivors who had relapsed in the first 5 years following their diagnosis had an almost 3 -fold increased risk of death $\left(\mathrm{HR}_{\mathrm{adj}}=2.9,95 \% \mathrm{CI}, 1.9\right.$ 4.5), compared to those who had not. RT was also associated with a 2-fold increased risk $\left(\mathrm{HR}_{\mathrm{adj}}=2.0,95 \% \mathrm{CI}, 1.3-3.1\right)$, compared to those who had not received RT.

Compared with survivors of lymphoma, survivors of both melanoma and carcinoma had decreased risks for the development of an SMN ( $\mathrm{HR}_{\mathrm{adj}}=0.3,95 \% \mathrm{CI}=0.1-0.6$; $\mathrm{HR}_{\mathrm{adj}}=0.2,95 \% \mathrm{CI}=0.1-0.5$, resp.) (Table 5). The other independent predictor for SMN, in addition to type of original diagnosis, was exposure to $\mathrm{RT}\left(\mathrm{HR}_{\mathrm{adj}}=2.0,95 \%\right.$ $\mathrm{CI}=1.1-3.7)$.

\section{Discussion}

To our knowledge, this is the first study to evaluate the risk of mortality and SMN, and the effects of disease-related characteristics, in a cohort of survivors of young adult cancer. We found increased risks of death and SMN in the survivors compared with the general population. Not surprisingly, survivors with recurrence of their original cancer and those with a second malignancy had higher mortality. Survivors with an original diagnosis of lymphoma had an elevated risk for the development of an SMN. Exposure to RT was directly associated with an increased risk of death and SMN. Use of linked population-based registries, health records, and vital statistics data in this study allows for essentially complete cases and ascertainment of outcomes over many years, and assessment of the impact of demographic and clinical factors on risk.

Our analysis indicated a six-fold increase in mortality among 5 -year survivors of young adult cancer. This estimate is lower than the SMRs identified from studies in childhood cancer survivors, ranging from 7.5 to $10.8[5,6,10,20$ 22 . These studies all focused on survivors of younger age than our study, with diagnoses $0-15$ years old in British study [20], 0-20 years old in the Nordic study [5], and $0-21$ years old in the CCSS study $[6,21]$. This difference in SMR is likely caused by the lower proportion of some types of cancer diagnosed in young adulthood compared to childhood, such as CNS tumor, which have a poor prognosis when diagnosed at younger ages [21]. It is also possible that cancer therapy delivered at an early age when the young person is experiencing a singular amount of growth and development may have a profound impact later on.

Studies of childhood cancer found that recurrence or progression of original cancer was the leading cause of death for survivors $[5,6,10,20,21]$. Meanwhile, several studies raised a concern that the cause of death was potentially related to the length of follow-up [5, 20, 23]. A study among $15+$ year survivors of childhood and adolescent cancers reported second malignancies to be the leading cause of death [23]. Our study identified the original cancer to be the leading cause of death for survivors with less than 20 years follow-up, and noncancer-related death to be the leading cause of death amongst survivors with more than 20 years of follow-up, followed by second malignancies, representing $43.3 \%$ and $33.3 \%$ of deaths respectively. Taken together, these results suggest that the longer time the patient has survived, the less likely the survivor is to die from the original cancer.

In this study, we found that female survivors of young adult cancer were at increased risk for the development of breast cancer, particularly those previously treated for the Hodgkin lymphoma. Several previous studies conducted in childhood cancer survivors have reported that thoracic RT is associated with an increased risk of breast cancer, and it is estimated that at least $12 \%$ to $20 \%$ of women exposed to moderate to high dose thoracic RT would go on to develop breast cancer [24-27]. Age at treatment did not appear to 
TABLE 4: Hazard ratio of mortality for all causes of death.

\begin{tabular}{|c|c|c|}
\hline & \multicolumn{2}{|c|}{ All causes of death $(N=138)$} \\
\hline & Unadjusted HR (95\% CI) & Adjusted HR (95\% CI) \\
\hline \multicolumn{3}{|l|}{ Sex ${ }^{\mathrm{a}}$} \\
\hline Female & 1 & 1 \\
\hline Male & $1.9(1.4-2.7)^{*}$ & $1.7(1.2-2.4)^{*}$ \\
\hline \multicolumn{3}{|l|}{ Type of original cancer ${ }^{b}$} \\
\hline Lymphoma & 1 & 1 \\
\hline Central nervous system tumors & $3.1(1.9-5.2)^{*}$ & $3.4(2.1-5.7)^{*}$ \\
\hline Soft tissue sarcomas & $1.1(0.6-2.2)$ & $1.2(0.6-2.3)$ \\
\hline Germ cell tumor & $0.3(0.2-0.7)^{*}$ & $0.4(0.2-0.7)^{*}$ \\
\hline Melanoma & $0.5(0.3-0.9)^{*}$ & $0.7(0.4-1.2)$ \\
\hline Carcinoma (except of skin) & $0.4(0.2-0.6)^{*}$ & $0.5(0.3-0.8)^{*}$ \\
\hline Others $^{\mathrm{c}}$ & $1.0(0.5-1.9)$ & $1.1(0.6-2.1)$ \\
\hline \multicolumn{3}{|l|}{ Diagnosis period $^{\mathrm{d}}$} \\
\hline 1970-1979 & 1 & 1 \\
\hline 1980-1989 & $0.7(0.4-1.0)$ & $0.6(0.4-0.9)^{*}$ \\
\hline 1990-1995 & $0.9(0.5-1.5)$ & $0.9(0.5-1.4)$ \\
\hline \multicolumn{3}{|l|}{ Relapse $<5$ years after diagnosis ${ }^{\mathrm{e}}$} \\
\hline No & 1 & 1 \\
\hline Yes & $4.1(2.7-6.3)^{*}$ & $2.9(1.9-4.5)^{*}$ \\
\hline \multicolumn{3}{|l|}{ Having chemotherapy ${ }^{\mathrm{f}}$} \\
\hline No & 1 & 1 \\
\hline Yes & $1.1(0.8-1.6)$ & $0.9(0.6-1.5)$ \\
\hline \multicolumn{3}{|l|}{ Having radiation ${ }^{\mathrm{f}}$} \\
\hline No & 1 & 1 \\
\hline Yes & $2.7(2-3.8)^{*}$ & $2.0(1.3-3.1)^{*}$ \\
\hline \multicolumn{3}{|l|}{ Having surgery ${ }^{f}$} \\
\hline No & 1 & 1 \\
\hline Yes & $0.6(0.5-0.9)^{*}$ & $1.3(0.9-1.9)$ \\
\hline \multicolumn{3}{|c|}{$\begin{array}{l}{ }^{*} P<0.05 . \\
\text { a Multivariate analyses adjusted for type of original cancer, diagnosis period, and relapse }<5 \text { years after diagnosis. } \\
\text { b Multivariate analyses adjusted for sex, diagnosis period, and relapse }<5 \text { years after diagnosis. } \\
{ }^{c} \text { Other types of original cancers include leukemia, bone tumor, and miscellaneous-specified tumor. } \\
\text { d Multivariate analyses adjusted for sex, type of original cancer, and relapse }<5 \text { years after diagnosis. } \\
{ }^{\mathrm{e}} \text { Multivariate analyses adjusted for sex, type of original cancer, and diagnosis period. } \\
{ }^{\mathrm{f}} \text { Multivariate analyses adjusted for sex, type of original cancer, diagnosis period, and relapse }<5 \text { years after diagnosis. }\end{array}$} \\
\hline
\end{tabular}

be significantly associated with the risk of subsequent breast cancer in most studies [28-30]. One study reported that the risk of breast cancer was not significant in girls treated between age 5-9 years old, but the risk became significantly increased in girls treated after the age of 10 [31]. The possible explanations for these findings include that proliferating and developing breast tissue, rather than prepubertal breast tissues, may be more sensitive to the effect of radiation; familial cancer syndromes may have important effect in breast cancer risk; the length of follow-up may not be sufficient for developing breast cancer [32]. Our findings are consistent with the recommendation for early and increased breast cancer screening in women exposed to moderate to high dose thoracic RT as part of a diagnosis of cancer in childhood and young adulthood, starting at 25 years of age or 8 years after radiation [33-35].
It is well known that chemotherapy and RT are associated with increased risks of SMN [24, 25, 36, 37]. In our study, we found 6-fold excess risks of SMN associated with chemotherapy and RT. 30\% (19/62 cases) of SMNs developed in the RT exposure field, whereas 23\% (14/62 cases) developed SMNs without RT and chemotherapy, suggesting that genetic or other factors may also play an important role in the occurrence of these SMNs.

Although the availability of administrative dataset and tumor treatment information on the majority of subjects and the long follow-up time period were strengths of the study, our study was limited by relatively small numbers of cases, which ruled out the possibility of assessing treatmentrelated dose-response for mortality and SMN risk. Other limitations included the missing data on treatment modality (approximately $28 \%$ of cases with missing chemotherapy 
TABLE 5: Hazard ratio of SMN.

\begin{tabular}{|c|c|c|}
\hline & \multicolumn{2}{|c|}{$\operatorname{SMN}(N=62)$} \\
\hline & Unadjusted HR (95\% CI) & Adjusted HR (95\% CI) \\
\hline \multicolumn{3}{|l|}{$\operatorname{Sex}^{\mathrm{a}}$} \\
\hline Female & 1 & 1 \\
\hline Male & $0.9(0.5-1.5)$ & $0.7(0.4-1.2)$ \\
\hline \multicolumn{3}{|l|}{ Type of original cancer ${ }^{\mathrm{b}}$} \\
\hline Lymphoma & 1 & 1 \\
\hline Germ cell tumor & $0.4(0.2-0.9)^{*}$ & $0.5(0.2-1.1)$ \\
\hline Melanoma & $0.3(0.1-0.7)^{*}$ & $0.3(0.1-0.6)^{*}$ \\
\hline Carcinoma (except of skin) & $0.3(0.1-0.5)^{*}$ & $0.2(0.1-0.5)^{*}$ \\
\hline Others $^{\mathrm{c}}$ & $0.3(0.1-0.7)^{*}$ & $0.3(0.1-0.7)^{*}$ \\
\hline \multicolumn{3}{|l|}{ Diagnosis period $^{\mathrm{d}}$} \\
\hline 1970-1979 & 1 & 1 \\
\hline 1980-1989 & $1.5(0.8-2.7)$ & $1.5(0.8-2.8)$ \\
\hline 1990-1995 & $1.8(0.6-5.1)$ & $1.7(0.6-5)$ \\
\hline \multicolumn{3}{|l|}{ Relapse $<5$ years after diagnosis ${ }^{\mathrm{e}}$} \\
\hline No & 1 & 1 \\
\hline Yes & $1.9(0.8-4.3)$ & $1.2(0.5-2.9)$ \\
\hline \multicolumn{3}{|l|}{ Having chemotherapy ${ }^{f}$} \\
\hline No & 1 & 1 \\
\hline Yes & $1.9(1.1-3.3)^{*}$ & $1.3(0.7-2.5)$ \\
\hline \multicolumn{3}{|l|}{ Having radiation $^{\mathrm{f}}$} \\
\hline No & 1 & 1 \\
\hline Yes & $2.9(1.8-4.9)^{*}$ & $2.0(1.1-3.7)^{*}$ \\
\hline \multicolumn{3}{|l|}{ Having surgery ${ }^{f}$} \\
\hline No & 1 & 1 \\
\hline Yes & $0.8(0.5-1.5)$ & $1.4(0.7-2.7)$ \\
\hline
\end{tabular}

${ }^{*} P<0.05$.

${ }^{a}$ Multivariate analyses adjusted for type of original cancer, diagnosis period, and relapse $<5$ years after diagnosis.

${ }^{b}$ Multivariate analyses adjusted for sex, diagnosis period, and relapse $<5$ years after diagnosis.

${ }^{\mathrm{c}}$ Other types of original cancers include leukemia, CNS, bone tumor, soft tissue sarcoma, and miscellaneous-specified tumor.

${ }^{\mathrm{d}}$ Multivariate analyses adjusted for sex, type of original cancer, and relapse $<5$ years after diagnosis.

${ }^{\mathrm{e}}$ Multivariate analyses adjusted for sex, type of original cancer, and diagnosis period.

${ }^{\mathrm{f}}$ Multivariate analyses adjusted for sex, type of original cancer, diagnosis period, and relapse $<5$ years after diagnosis.

and surgery), and the unknown information on vital status and SMN, due to events that may have occurred outside of Canada.

Even after more than 20 years follow-up, the majority of young adult cancer survivors have just reached middle age. It is possible that, with longer follow-up, the risks of mortality and SMN may increase together with other long-term late health effects. This study emphasizes the importance of screening for late effects such as SMN leading to mortality, and the development of prevention and intervention strategies to address chronic health problems in these survivors.

\section{Conclusion}

In conclusion, our cohort study shows that young adult cancer survivors experience elevated risks of mortality and SMN over many years compared with the general population. Although the increased risks of mortality and SMN were generally observed across all demographic and treatment-related groups, the risk was highest in survivor groups characterized by a later time period of diagnosis, an original diagnosis of lymphoma and receipt of radiation therapy. These elevated risks suggest that future efforts should focus on the development of screening programs for early detection of late effects and strategies to help prevent these significant long-term health problems among survivors of young adult cancer.

\section{Conflict of Interests}

The authors indicate that there is no potential conflict of interests to declare.

\section{Acknowledgments}

The authors gratefully acknowledge funding from the Canadian Cancer Society (CCS) Research Institute, and the CCS (BC and Yukon Division) for CAYACS Program support (PPG no. 16000 and no. 16001); the BC Cancer Registry and 
BC Cancer Agency for their cooperation in allowing access, use, and linkage of the data to support this program.

\section{References}

[1] N. Howlader, N. N. A, M. Krapcho et al., Eds., "SEER Cancer Statistics Review, 1975-2008," National Cancer Institute, Bethesda, Md, USA, http://seer.cancer.gov/csr/1975_2008/.

[2] Cancer Care Ontario, "Cancer in Young Adults in Canada," Tech. Rep., Toronto, Canada, 2006.

[3] A. Bleyer and R. Barr, Cancer in Adolescents and Young Adults, Springer, Berlin, Germany, 1st edition, 2007.

[4] E. Woodward, M. Jessop, A. Glaser, and D. Stark, "Late effects in survivors of teenage and young adult cancer: does age matter?" Annals of Oncology, vol. 22, no. 12, Article ID mdr044, pp. 2561-2568, 2011.

[5] T. R. Möller, S. Garwicz, L. Barlow et al., "Decreasing late mortality among five-year survivors of cancer in childhood and adolescence: a population-based study in the Nordic countries," Journal of Clinical Oncology, vol. 19, no. 13, pp. 31733181, 2001.

[6] A. C. Mertens, Y. Yasui, J. P. Neglia et al., "Late mortality experience in five-year survivors of childhood and adolescent cancer: the childhood cancer survivor study," Journal of Clinical Oncology, vol. 19, no. 13, pp. 3163-3172, 2001.

[7] A. C. Mertens, Q. Liu, J. P. Neglia et al., "Cause-specific late mortality among 5-year survivors of childhood cancer: the childhood cancer survivor study," Journal of the National Cancer Institute, vol. 100, no. 19, pp. 1368-1379, 2008.

[8] M. C. Cardous-Ubbink, R. C. Heinen, N. E. Langeveld et al., "Long-term cause-specific mortality among five-year survivors of childhood cancer," Pediatric Blood and Cancer, vol. 42, no. 7, pp. 563-573, 2004.

[9] E. Dama, G. Pastore, M. L. Mosso et al., "Late deaths among five-year survivors of childhood cancer. A population-based study in Piedmont Region, Italy," Haematologica, vol. 91, no. 8, pp. 1084-1091, 2006.

[10] A. C. MacArthur, J. J. Spinelli, P. C. Rogers, K. J. Goddard, Z. U. Abanto, and M. L. McBride, "Mortality among 5-year survivors of cancer diagnosed during childhood or adolescence in British Columbia, Canada," Pediatric Blood and Cancer, vol. 48, no. 4, pp. 460-467, 2007.

[11] M. M. Hawkins, J. E. Kingston, and L. M. Kinnier Wilson, "Late deaths after treatment for childhood cancer," Archives of Disease in Childhood, vol. 65, no. 12, pp. 1356-1363, 1990.

[12] C. M. Robertson, M. M. Hawkins, and J. E. Kingston, "Late deaths and survival after childhood cancer: implications for cure," BMJ, vol. 309, no. 6948, pp. 162-166, 1994.

[13] H. Soliman and S. V. Agresta, "Current issues in adolescent and young adult cancer survivorship," Cancer Control, vol. 15, no. 1, pp. 55-62, 2008.

[14] M. L. McBride, P. C. Rogers, S. B. Sheps et al., "Childhood, adolescent, and young adult cancer survivors research program of British Columbia: objectives, study design, and cohort characteristics," Pediatric Blood and Cancer, vol. 55, no. 2, pp. 324-330, 2010.

[15] R. D. Barr, E. J. Holowaty, and J. M. Birch, "Classification schemes for tumors diagnosed in adolescents and young adults," Cancer, vol. 106, no. 7, pp. 1425-1430, 2006.

[16] World Health Organization, "International classification of diseases: manual of the international classification of diseases, injuries and causes of death," 9th revision, WHO, Geneva, Switzerland, 1979.
[17] A. Fritz et al., International Classification of Diseases for Oncology, World Health Organization, Geneva, Switzerland, 3rd edition, 2000.

[18] J. P. Fine and R. J. Gray, "A proportional hazards model for the subdistribution of a competing risk," Journal of the American Statistical Association, vol. 94, no. 446, pp. 496-509, 1999.

[19] R. J. A. Little and D. B. Rubin, Statistical Analysis with Missing Data, John Wiley \& Sons, NJ, USA, 2nd edition, 2002.

[20] R. C. Reulen, D. L. Winter, C. Frobisher et al., "Long-term cause-specific mortality among survivors of childhood cancer," Journal of the American Medical Association, vol. 304, no. 2, pp. 172-179, 2010.

[21] G. T. Armstrong, Q. Liu, Y. Yasui et al., "Late mortality among 5-year survivors of childhood cancer: a summary from the childhood cancer survivor study," Journal of Clinical Oncology, vol. 27, no. 14, pp. 2328-2338, 2009.

[22] T. R. Möller, S. Garwicz, R. Perfekt et al., "Late mortality among five-year survivors of cancer in childhood and adolescence: differences between the Nordic countries," Acta Oncologica, vol. 43, no. 8, pp. 711-718, 2004.

[23] S. C. W. Lawless, P. Verma, D. M. Green, and M. C. Mahoney, "Mortality experiences among 15+ year survivors of childhood and adolescent cancers," Pediatric Blood and Cancer, vol. 48, no. 3, pp. 333-338, 2007.

[24] J. P. Neglia, D. L. Friedman, Y. Yasui et al., "Second malignant neoplasms in five-year survivors of childhood cancer: childhood cancer survivor study," Journal of the National Cancer Institute, vol. 93, no. 8, pp. 618-629, 2001.

[25] M. C. Cardous-Ubbink, R. C. Heinen, P. J. M. Bakker et al., "Risk of second malignancies in long-term survivors of childhood cancer," European Journal of Cancer, vol. 43, no. 2, pp. 351-362, 2007.

[26] A. C. MacArthur, J. J. Spinelli, P. C. Rogers, K. J. Goddard, N. Phillips, and M. L. McBride, "Risk of a second malignant neoplasm among 5-year survivors of cancer in childhood and adolescence in British Columbia, Canada," Pediatric Blood and Cancer, vol. 48, no. 4, pp. 453-459, 2007.

[27] C. L. Wilson, R. J. Cohn, K. A. Johnston, and L. J. Ashton, "Late mortality and second cancers in an Australian cohort of childhood cancer survivors," Medical Journal of Australia, vol. 193, no. 5, pp. 258-261, 2010.

[28] S. Bhatia, Y. Yasui, L. L. Robison et al., "High risk of subsequent neoplasms continues with extended follow-up of childhood Hodgkin's disease: report from the Late Effects Study Group," Journal of Clinical Oncology, vol. 21, no. 23, pp. 4386-4394, 2003.

[29] L. B. Travis, D. A. Hill, G. M. Dores et al., "Breast cancer following radiotherapy and chemotherapy among young women with hodgkin disease," Journal of the American Medical Association, vol. 290, no. 4, pp. 465-475, 2003.

[30] P. D. Inskip, L. L. Robison, M. Stovall et al., "Radiation dose and breast cancer risk in the childhood cancer survivor study," Journal of Clinical Oncology, vol. 27, no. 24, pp. 3901-3907, 2009.

[31] L. B. Kenney, Y. Yasui, P. D. Inskip et al., "Breast cancer after childhood cancer: a report from the Childhood Cancer Survivor Study," Annals of Internal Medicine, vol. 141, no. 8, pp. 590-597, 2004.

[32] A. K. Ng, L. B. Kenney, E. S. Gilbert, and L. B. Travis, "Secondary malignancies across the age spectrum," Seminars in Radiation Oncology, vol. 20, no. 1, pp. 67-78, 2010.

[33] W. Landier, S. Bhatia, D. A. Eshelman et al., "Development of risk-based guidelines for pediatric cancer survivors: the Children's Oncology Group Long-Term Follow-Up Guidelines 
from the Children's Oncology Group Late Effects Committee and Nursing Discipline," Journal of Clinical Oncology, vol. 22, no. 24, pp. 4979-4990, 2004.

[34] K. C. Oeffinger, J. S. Ford, C. S. Moskowitz et al., "Breast cancer surveillance practices among women previously treated with chest radiation for a childhood cancer," Journal of the American Medical Association, vol. 301, no. 4, pp. 404-414, 2009.

[35] "Long-term follow-up guidelines for survivors of childhood, adolescent, and young adult cancer," Children's Oncology Group, 2008.

[36] M. Bassal, A. C. Mertens, L. Taylor et al., "Risk of selected subsequent carcinomas in survivors of childhood cancer: a report from the childhood cancer survivor study," Journal of Clinical Oncology, vol. 24, no. 3, pp. 476-483, 2006.

[37] H. C. Jenkinson, M. M. Hawkins, C. A. Stiller, D. L. Winter, H. B. Marsden, and M. C. G. Stevens, "Long-term populationbased risks of second malignant neoplasms after childhood cancer in Britain," British Journal of Cancer, vol. 91, no. 11, pp. 1905-1910, 2004. 


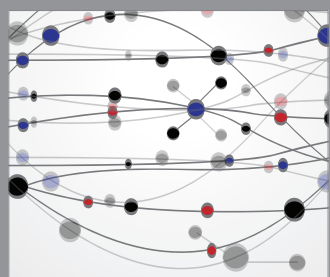

The Scientific World Journal
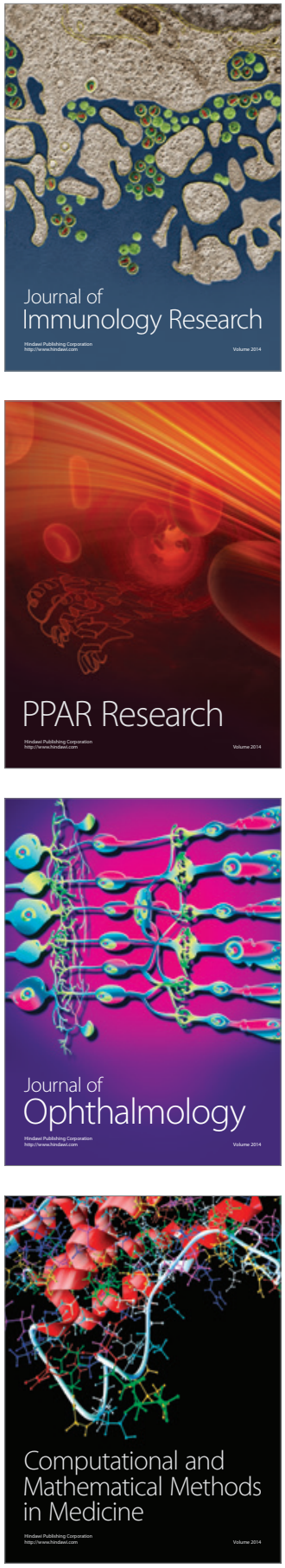

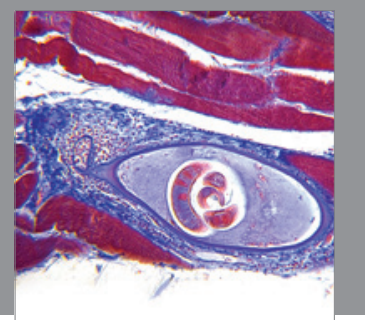

Gastroenterology

Research and Practice
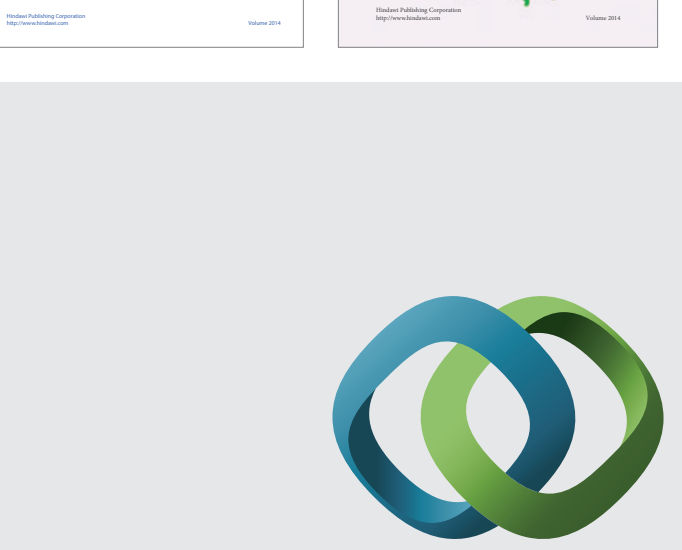

\section{Hindawi}

Submit your manuscripts at

http://www.hindawi.com
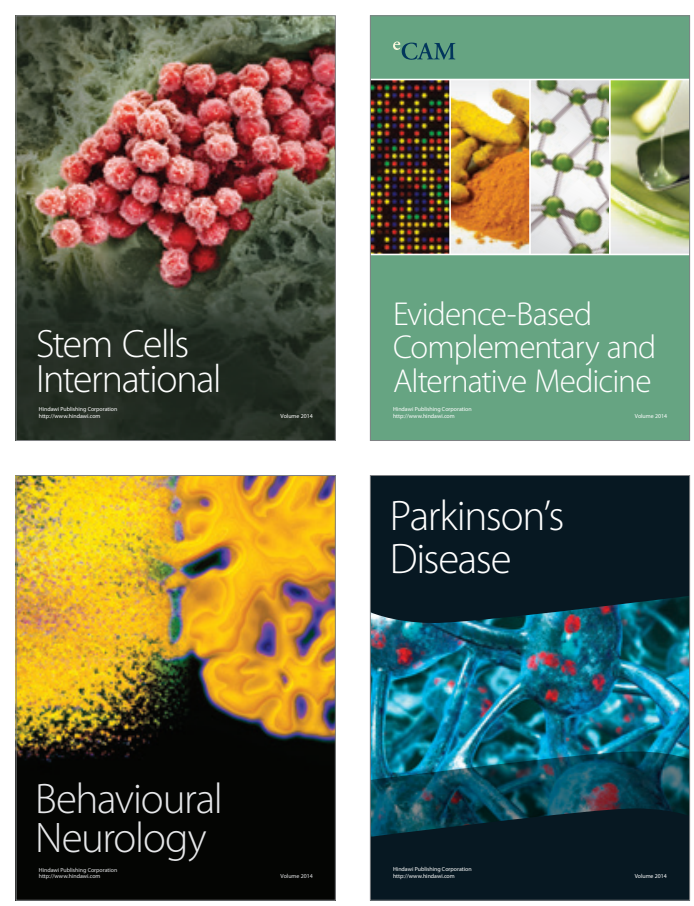

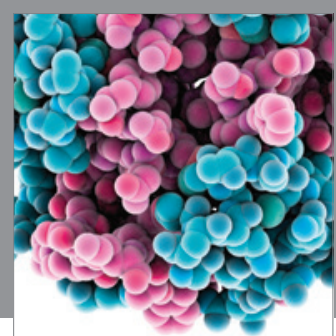

Journal of
Diabetes Research

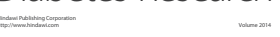

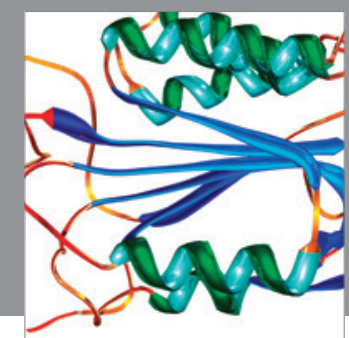

Disease Markers
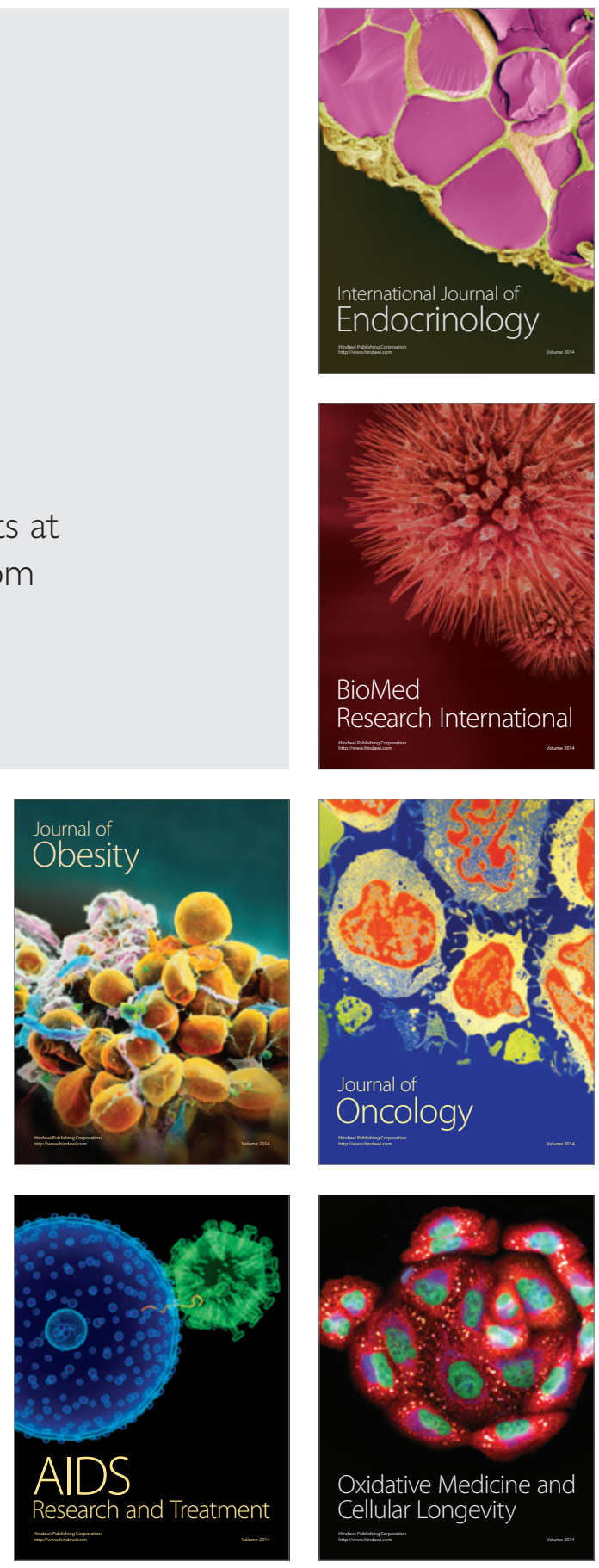